\title{
Was hilft am besten bei Kopfgneis?
}

\author{
Dr. Markus Schneider, Facharzt für Kinder- und Jugendmedizin, Bad Rappenau
}

\section{Die Begriffe Kopfgneis und Milchschorf werden irrtümlich häufig synonym verwendet. Beide bezeichnen schuppige Auflagerungen auf der Kopfhaut bei Säuglingen und Kleinkindern. Zwar besteht aus medizinischer Sicht keine Behandlungsnotwendigkeit, doch wünschen viele Eltern eine einfache, sichere und effektive Möglichkeit, die Schup- pen zu entfernen. In einer neuen Studie erweist sich die Anwendung einer modernen Oleogel-Formulierung gegenüber der Behandlung mit Olivenöl überlegen.}

\section{Terminologie}

Kopfgneis gilt allgemein gesprochen als eine Manifestationsform der seborrhoischen Säuglingsdermatitis. Davon zu unterscheiden ist der Begriff Milchschorf (Crusta lactea), der im allgemeinen Sprachgebrauch oft synonym verwendet wird.

Der Begriff Gneis oder Kopfgneis wird bei kleieförmigen, fettigen, massiven Schuppungen, meist mit Sitz in der Mitte des Vorderkopfes mit stellenweisem Übergreifen auf die Stirn verwendet. In aller Regel entsteht dieser auf der Kopfhaut bei Säuglingen im Alter zwischen 2 Wochen und 6 Monaten, die Häufigkeit wird mit 3-5\% aller Säuglinge angegeben [1]. Die Hautveränderungen klingen meist gegen Ende des ersten bzw. Anfang des zweiten Lebensjahres ab, können auch bis zum 10. Lebensjahr in unterschiedlicher Schwere weiterbestehen [2].

Die Aktivität der Talgdrüsen und die Talgproduktion sind hormon- und altersabhängig. Neugeborene haben große Talgdrüsen und eine hohe Sebumsekretionsrate. Einfluss darauf haben Androgene, die als Rückstände mütterlicher Hormone in den ersten Lebensmonaten im Körper des Säuglings nachweisbar sind und zu einer verstärkten Talgproduktion beitragen. Im Kindesalter sinkt die Talgdrüsenaktivität auf ein Minimum und steigt mit Beginn der Pubertät wieder an $[1,3,4,5]$. Beim Kopfgneis in seiner im Allgemeinen eher leichten Ausprägung handelt es sich nicht um eine bedrohliche, aber um eine von den Eltern oftmals als sehr lästig empfundene Störung [1, 2, 3].

Abzugrenzen vom Kopfgneis ist eine Frühmanifestation der atopischen Dermatitis, die sich jedoch meist erst im 3.-5. Lebensmonat entwickelt, stärker entzündliche Veränderungen zeigt, teils

Tab. 1 Eigenschaften von Kopfgneis und Milchschorf.

\begin{tabular}{|c|c|c|}
\hline & Kopfgneis & Milchschorf \\
\hline Manifestationsform & Seborrhoische Dermatitis & Atopische Dermatitis \\
\hline Auftreten & $\begin{array}{l}\text { 2. Lebenswoche bis } 6 \text {. Lebens- } \\
\text { monat }\end{array}$ & 3.-5. Lebensmonat \\
\hline Dauer & $\begin{array}{l}\text { verschwindet meist zwischen } \\
\text { dem 1. und 2. Lebensjahr }\end{array}$ & häufig auch mehrere Jahre \\
\hline Verlauf & meist spontanes Verschwinden & $\begin{array}{l}\text { oft Chronifizierung im } \\
\text { Rahmen einer atopischen } \\
\text { Dermatitis }\end{array}$ \\
\hline Erscheinungsbild & $\begin{array}{l}\text { fettige, kleieförmige, gelb- } \\
\text { braune oft festanhaftende } \\
\text { Schuppenschicht auf Kopfhaut } \\
\text { und Stirn }\end{array}$ & $\begin{array}{l}\text { harte Schuppen, Verkrustun- } \\
\text { gen, teils mit entzündlichen } \\
\text { Veränderungen auf Kopfhaut }\end{array}$ \\
\hline Juckreiz & selten & häufig \\
\hline
\end{tabular}

mit harten Schuppen und Verkrustungen und häufig mit relevantem Juckreiz einhergeht [1, 3, 5]. Die gelblich, fettig glänzenden, dicken, oft fissurierten krustösen Plaques auf der Kopfhaut erinnern an das Aussehen von angebrannter Milch, weshalb dieser Zustand häufig mit dem Begriff Milchschorf bezeichnet wird. Tab. 1 zeigt eine tabellarische $\mathrm{Zu}$ sammenfassung der Eigenschaften von Kopfgneis und Milchschorf. Im weiteren Text beziehen wir uns nun ausschließlich auf die Behandlung von Kopfgneis. 


\section{Therapieoptionen}

Strenggenommen ist aus medizinischer Sicht eine Behandlungsnotwendigkeit bei Kopfgneis meist nicht gegeben. Es ist jedoch der nachdrückliche Wunsch vieler Eltern, die Schuppen einfach, sicher und effektiv zu entfernen. Bei ausgeprägtem Kopfgneis können der in diesen Fällen selten auftretende Juckreiz bzw. die übermäßige Besiedlung mit Hefepilzen und der damit verbundene unangenehme Geruch Therapieindikationen darstellen.

Obwohl Kopfgneis häufig vorkommt, war bisher eine rasche Beseitigung der verstärkten Schuppung eher problematisch. Die Datenlage bezüglich erfolgreicher Maßnahmen zur Beseitigung von Gneis ist sehr beschränkt. Es fehlen aussagekräftige Studien.

Empfohlen wird bislang häufig das Auftragen von Pflegeprodukten wie Oliven- oder Mandelöl, teils unter einer Kopfkappe und über Nacht, um ein Aufweichen der Krusten zu erreichen. Anschließend ist der Kopf mit Shampoo abzuwaschen, gegebenenfalls unterstützt durch vorsichtiges Reiben. Jedoch ist die Wirksamkeit dieser Anwendungen häufig nicht ausreichend für eine vollständige Entfernung.

Eine weitere Steigerung der Maßnahmen, insbesondere bei dicken festanhaftenden Schuppen, ist der Einsatz fett-feuchter Wickel. Neben einer häufig nicht ausreichenden Wirksamkeit ist die überwiegend über Nacht durchgeführte Prozedur umständlich und für den kleinen Säugling belastend. Ähnliches gilt für den Einsatz von Silikonöl/Dimeticon. Dieses wird bei einer Einwirkzeit von mindestens drei Stunden und gegebenenfalls über Nacht ein- bis dreimal angewendet. Hierzu existiert aktuell eine Studie, die auf eine Wirksamkeit hindeutet [6].

Der (z.B. auf Informationsseiten im Internet) auch heute noch empfohlene Einsatz von Salicylsäure verbietet sich aufgrund der Toxizität der Substanz in den ersten Lebensmonaten. Arzneiliche Ansätze sind ebenfalls wenig hilfreich. Antimykotisch wirksame Shampoos sind beim adulten seborrhoischen Ekzem ein zentraler Therapiebestandteil, aber für die Anwendung im Säuglingsalter fehlt eine Zulassung. Zudem spielen Pilzinfektionen beim Gneis im Säuglingsalter äthiologisch keine Rolle. Topische Steroide bei stärker entzündlichen Hautreaktionen werden insbesondere aufgrund des Nebenwirkungsprofils sehr kontrovers gesehen und sollten bei dieser unkomplizierten Befindlichkeitsstörung im Säuglingsalter nicht ohne Not zum Einsatz kommen [1].

Des Weiteren steht zur Schuppenentfernung bei Kopfgneis seit 2013 eine Oleogel-Formulierung (Macadamia-Öl, Jojoba-Öl, Olivenöl, Glycerin, Triglyceride und Mineralien) als Kosmetikum auf dem Markt zur Verfügung [1, 2, 7]. Mit der Rezeptur wurde die Grundidee, talgige Beläge mit Öl zu lösen, weiterent- 
wickelt. Nach dem Prinzip „Gleiches in Gleichem lösen“ löst das Oleogel die öligen und somit fettlöslichen Schuppen von der Kopfhaut. Durch Zugabe von Wasser entsteht dann eine milchige Emulsion, die sich abspülen lässt. Die Anwendung des Oleogels ist ab der 3. Lebenswoche empfohlen [7].

\section{Randomisierte Vergleichs- studie zur Schuppen- entfernung bei Kopfgneis}

Ziel der vorliegenden Studie war es, die Überlegenheit der Schuppenentfernung bei Kopfgneis durch ein modernes Oleogel gegenüber Olivenöl zu zeigen und so die bisherigen guten Erfahrungen wissenschaftlich zu bestätigen. Das eingesetzte Oleogel befindet sich als Kosmetikum unter dem Namen BabyBene ${ }^{\circledR}$ Gel (Macadamia-Öl, Jojoba-Öl, Olivenöl, Glycerin, Triglyceride und Mineralien; Pädia $\mathrm{GmbH}$, Heppenheim) auf dem Markt. Insgesamt wurden 39 Kinder im Alter von 3 bis 17 Monaten mit klinisch bestätigtem Kopfgneis untersucht und behandelt. Die Studienteilnehmer erhielten randomisiert entweder das Oleogel (20 Patienten) oder Olivenöl (kosmetische Qualität, 19 Patienten). Die Anwendung wurde in einem der drei Prüfzentren (eine Kinderklinik und zwei Kinderarztpraxen) durchgeführt. Dazu wurde das entsprechende Produkt aufgetragen und für 2 bis 3 Minuten einmassiert. Die weitere Einwirkzeit betrug 30 Minuten. Danach wurde tropfenweise Wasser auf das Areal gegeben und nach weiteren $5 \mathrm{Mi}$ nuten der Kopf mit lauwarmen Wasser und einem Schwamm unter reibenden Bewegungen abgespült. Der Anwendungserfolg, die Anwendung an sich sowie Wirkung und Gesamteindruck wurden direkt nach der Anwendung des Testproduktes durch den Arzt und die Eltern mittels Schulnotenskala bewertet. Zusätzlich bewertete der Arzt die prozentuale Schuppenentfernung. Im Falle eines nicht zufriedenstellenden Ergebnisses nach Olivenöl-Anwendung wurde den Eltern eine Folgeanwendung mit Oleogel angeboten. Die Folgeanwendung wurde dann ausschließlich durch die Eltern bewertet.

\section{Studienergebnisse}

In $100 \%$ der Anwendungen (20/20) führte das Oleogel zum Erfolg und ist damit einer Schuppenentfernung mit Olivenöl (26\% Erfolg, 5/19) signifikant überlegen ( $\mathrm{p}<0,0001, \mathrm{X}^{2}$-Test). Auch nach vorheriger, erfolgloser Olivenöl-Anwendung führte das Oleogel in allen Fällen (100\%) zum Erfolg (Abb. 1).

Die prozentuale Schuppenentfernung unter dem Oleogel ist der unter Olivenöl signifikant überlegen (Median $80 \%$ vs. $20 \%$, Mittelwert $79 \% \pm 14 \%$ vs. $27 \% \pm$ $26 \%, \mathrm{p}<0,0001$, Wilcoxon-Mann-Whitney/U-Test) (Abb. 2).

Abb. 3 zeigt eine beispielhafte Anwendung. Die Bewertung der Wirksamkeit durch Prüfer und Eltern ist in Tab. 2 zusammengefasst.

Die sehr guten Studienergebnisse belegen, dass das Oleogel eine effektive Schuppenentfernung im Fall von Kopfgneis beim Säugling ermöglicht. Die durchgehend signifikante Überlegenheit des Oleogels zeigt den großen Vorteil gegenüber einem Hausmittel wie Olivenöl (noch dazu in aller Regel in nicht medizinischer Qualität) mit mangelnder Wirksamkeit und in der Regel aufwendigem Anwendungsprozedere. Trotz der eher geringen Studienteilnehmerzahl, die sich aus der primär kosmetischen Behandlungsindikation ergibt, zeigen sich hier deutlich signifikante Ergebnisse. Insbe-
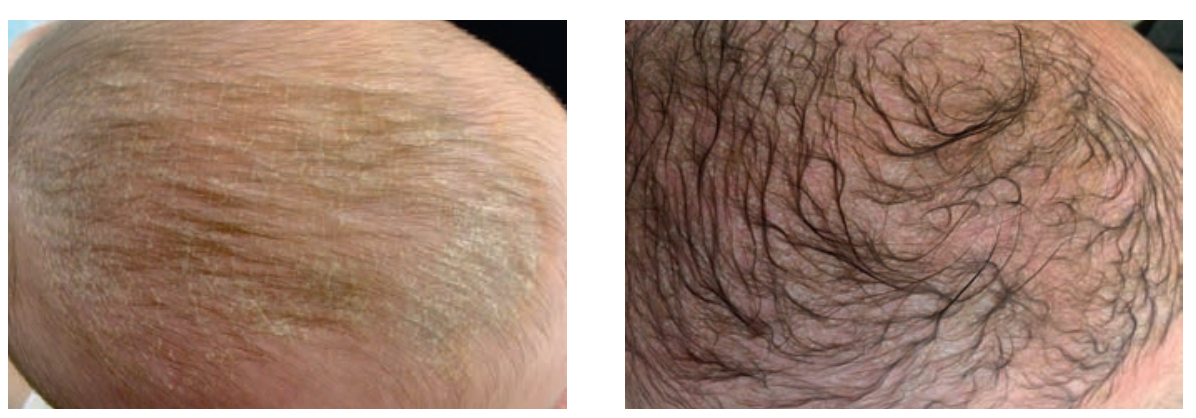

Abb. 3 Vorher - nachher: Anwendungserfolg nach einmaliger Anwendung von Oleogel mit 30 Minuten Einwirkzeit.

sondere der 100\%-ige Anwendungserfolg nach gescheiterter Olivenöl-Behandlung bestätigt die sehr gute Wirksamkeit auch für eine häusliche Anwendung durch die

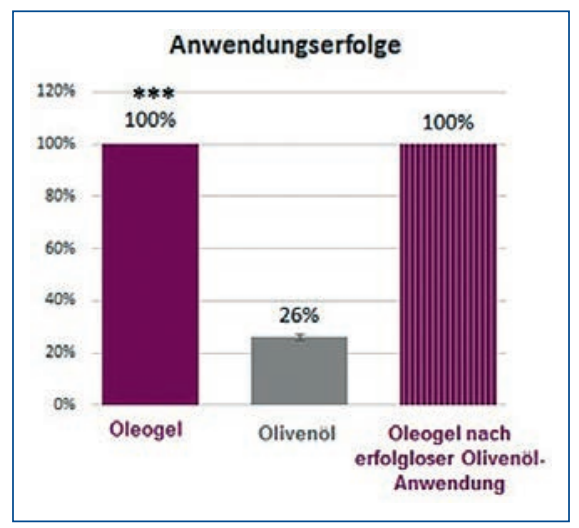

Abb. 1 Prozentualer Anwendungserfolg auf der Kopfhaut nach einer Anwendung ( $n=20$ für Oleogel, $n$ = 19 für Olivenöl, $\mathrm{n}=16$ für Oleogel nach gescheiterter Olivenöl-Anwendung); ${ }^{* * *} \mathrm{p}<0,0001$, $\mathrm{X}^{2}$-Test.

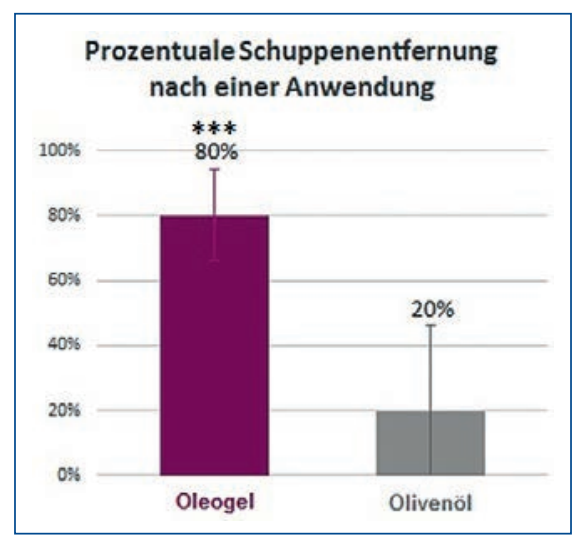

Abb. 2 Schuppenentfernung auf der Kopfhaut in \% (Median \pm Standardabweichungen) des initial betroffenen, behandelten Areals, bewertet durch den Prüfer nach einer Anwendung ( $n=20$ für Oleogel, $n=19$ für Olivenöl); ${ }^{* * *} \mathrm{p}<0,0001$, Wilcoxon-Test. 
Tab. 2 Bewertung durch Prüfer und Eltern.

\begin{tabular}{|l|l|l|l|}
\hline \multicolumn{1}{|l|}{ Gesamteindruck } & Zufriedenheit & Wirksamkeit \\
\hline Bewertung durch den Prüfer & & \\
\hline Oleogel & $\begin{array}{l}\text { Sehr gut } \\
(1 / 1,6 \pm 0,7)\end{array}$ & $\begin{array}{l}\text { Sehr gut } \\
(1 / 1,5 \pm 0,5)\end{array}$ & $\begin{array}{l}\text { Sehr gut } \\
(1 / 1,7 \pm 0,9)\end{array}$ \\
\hline Olivenöl & $\begin{array}{l}\text { Mangelhaft } \\
(5 / 4,4 \pm 1,3)\end{array}$ & $\begin{array}{l}\text { Ausreichend } \\
(4 / 4,0 \pm 1,4)\end{array}$ & $\begin{array}{l}\text { Mangelhaft } \\
(5 / 4,4 \pm 1,2)\end{array}$ \\
\hline Bewertung durch die Eltern & $\begin{array}{l}\text { Sehr gut } \\
(1 / 1,5 \pm 0,5)\end{array}$ & $\begin{array}{l}\text { Sehr gut } \\
(1 / 1,4 \pm 0,5)\end{array}$ & $(1 / 1,5 \pm 0,7)$ \\
\hline Oleogel & $\begin{array}{l}\text { Mangelhaft } \\
(5 / 4,5 \pm 1,3)\end{array}$ & $(5 / 3,9 \pm 1,6)$ & $\begin{array}{l}\text { Mangelhaft } \\
(5 / 4,4 \pm 1,3)\end{array}$ \\
\hline Olivenöl & &
\end{tabular}

Bewertung des Produktes durch Schulnoten von 1 = sehr gut bis 6 = ungenügend, angegeben als Median/ Mittelwert \pm Standardabweichungen, ( $n=20$ für Oleogel, $n=19$ für Olivenöl)

Eltern. Das Ausbleiben unerwünschter Wirkungen innerhalb der Oleogel-Gruppe belegt die Sicherheit der Anwendung.

\section{Zusammenfassung}

Zur Schuppenentfernung bei Kopfgneis im Säuglingsalter steht mit dem Oleogel ein effektives und sicheres Produkt mit einfacher Anwendung zur Verfügung. Das Oleogel ist zum Entfernen von Kopfschuppen bei Kopfgneis im Säuglingsalter gegenüber Olivenöl signifikant überlegen. Vergleichende Studien zu anderen auf dem Markt verfügbaren Produkten zur Anwendung bei Kopfgneis sollten folgen.

\section{Interessenkonflikte}

Dr. Schneider war wissenschaftlicher Leiter der SKBENE-Studie. Darüber hinaus bestehen keine Interessenkonflikte.

\section{Literatur}

1 Schneider M, Cremer H. Gneis und seine Therapieoptionen. Pädiatrie hautnah 2011; 23 (6): 459-460

2 Abeck D. So werden Kinderköpfe schuppenfrei. Der Allgemeinarzt 2010; 20: 34-35

3 Stögmann W. Gneis und Milchschorf. Pädiat Prax 2005; 66: 696-697
4 Weston WL, Lane AT, Morelli JG. Seborrhoic dermatitis. In: Color Textbook of Pediatric Dermatology. Mosby, Philadelphia 2006; 39-40

5 Krol A, Krafchik B. The differential diagnosis of atopic dermatitis in childhood. Dermatol Ther 2006; 19 (2): 73-82

6 Hengge U. Topical, non-medicated LOYON $^{\circledR}$ in facilitating the removal of scaling in infants and children with cradle cap: a proof-of-concept pilot study. Dermatol Ther 2014; 4: 221-232

7 InfectoPharm Arzneimittel. 2014. Packungsbeilage BabyBene ${ }^{\circledR}$ Gel

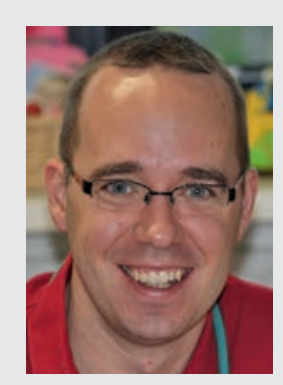

\section{Dr. med. Markus Schneider}

Facharzt für Kinder- und Jugendmedizin, Neugeborenen-Notarzt, Schwerpunkt Therapie von Hautkrankheiten im Kindes- \& Jugendalter/Hämangiome Praxis für Kinder- und Jugendmedizin Bad Rappenau Bahnhofstraße 9 74906 Bad Rappenau kinderhaut-schneider@gmx.de 